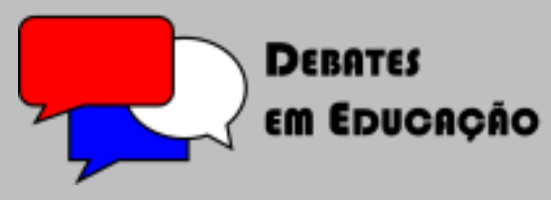

ISSN Eletrônico: 2175-6600

Vol. 9 | №. 19 | Set./Dez. | Ano 2017

\author{
David Wesley Amado Duarte \\ Instituto Federal de Educação, Ciência e \\ Tecnologia do Ceará (IFCE) \\ davidduarte@ifce.edu.br
}

\section{Q-MEMÓRIA: UM JOGO DIGITAL PARA O ESTUDO DE QUÍMICA}

\begin{abstract}
RESUMO
Este trabalho trata do desenvolvimento e avaliação pedagógica de um jogo da memória digital, denominado Q-Memória, que auxilia nos processos de ensino e de aprendizagem a partir da Tabela Periódica para alunos de Química do primeiro ano do ensino médio. O jogo foi desenvolvido na plataforma Unity $3 \mathrm{D}$, e avaliado por dezessete alunos do primeiro ano do ensino médio de um IF. Foi usada a metodologia pré-teste e pós-teste para avaliar o progresso da aprendizagem. O jogo possui oito níveis crescentes de dificuldade, baseados em uma sequência didática preparada com base nos conteúdos fundamentais de Química, apresentando os três tipos de aprendizagem significativa descritas por Ausubel, trazendo pares de cartas com conceitos associados, representações e proposições. Foi possível aferir a eficácia do Q-Memória na aprendizagem significativa de conceitos essenciais da disciplina. O modelo sequencial e hierárquico de apresentação das informações tornou possível uma maior estabilidade e clareza, levando os alunos a um melhor entendimento de conceitos mais complexos, a partir dos conceitos mais simples apresentados.
\end{abstract}

Palavras-chave: Tabela periódica. Linguagem Química. Jogos educativos digitais. Jogo da memória. Aprendizagem significativa.

\section{Q-MEMORIA: A DIGITAL GAME FOR THE CHEMISTRY STUDY}

\begin{abstract}
This work deals with the development and pedagogical evaluation of a game of digital memory, called Q-Memory, which assists in the teaching and learning processes from Periodic Table for chemistry students of the first year of high school. The game was developed on the Unity 3D platform and evaluated by seventeen first-year high school students from an IF. The pre-test and post-test methodology was used to evaluate the learning progress. The game has eight increasing levels of difficulty, based on a didactic sequence prepared based on the fundamental contents of Chemistry, presenting the three types of meaningful learning described by Ausubel, bringing pairs of letters with associated concepts, representations and propositions. It was possible to gauge efficacy of Q-Memory in meaningful learning of essential concepts of the discipline. The sequential and hierarchical model of presentation of information made possible greater stability and clarity, leading students to a better understanding of more complex concepts, from the simpler concepts presented.
\end{abstract}

Keywords: Periodic table. Chemical Language. Digital educational games. Memory game. Meaningful learning.

DOI: $10.28998 / 2175-6600.2017 v 9 n 19 p 155$ 


\section{RESUMO DA DISSERTAÇÃO}

Este trabalho trata do desenvolvimento e avaliação pedagógica de um jogo da memória digital, denominado Q-Memória, que auxilia nos processos de ensino e de aprendizagem a partir da Tabela Periódica para alunos de Química do primeiro ano do ensino médio. Professores preparam suas aulas de acordo com o modo como acreditam que os alunos aprendem. Isso pode ser verificado a partir das diversas teorias de aprendizagem, que se baseiam em diferentes princípios epistemológicos. As principais teorias de aprendizagem são objetivismo, behaviorismo, cognitivismo, construtivismo e conectivismo. Uma ramificação da teoria cognitivista é a Teoria da Aprendizagem Significativa. Essa Teoria, desenvolvida por David Ausubel, defende que os alunos aprendem baseados em conhecimentos prévios sobre o assunto, presentes na estrutura cognitiva, que podem receber novos conhecimentos que serão sedimentados sobre os primeiros. Este conhecimento é adquirido por um processo de aprendizagem baseado na aquisição, assimilação e retenção. O Q-Memória foi desenvolvido na plataforma Unity 3D. Para avaliação do jogo foram convidados dezessete alunos do primeiro ano do ensino médio de um IF. Foi aplicado um pré-teste para medir os conhecimentos anteriores dos alunos. Após o uso do jogo foi aplicado um pós-teste para avaliar o progresso da aprendizagem. Os testes foram comparados a partir da Análise de Diferença de Escores, com as notas do segundo teste sendo comparadas com as do primeiro. O jogo desenvolvido possui oito níveis crescentes de dificuldade, baseados em uma sequência didática preparada com base nos conteúdos fundamentais de Química. O jogo apresenta os três tipos de aprendizagem significativa descritas por Ausubel, trazendo pares de cartas com conceitos associados, representações e proposições. Após os experimentos, foi possível aferir a eficácia do Q-Memória na aprendizagem significativa de conceitos essenciais sobre Tabela Periódica. O modelo sequencial e hierárquico de apresentação das informações tornou possível uma maior estabilidade e clareza, levando os alunos a um melhor entendimento de conceitos mais complexos da disciplina, a partir dos conceitos mais simples apresentados.

\section{REFERÊNCIAS}

AGARWAL, Maya; SAHA, Shubhajit. Learning Chemistry Through Puzzle Based Game: Atoms to Molecule. INTERNATIONAL CONFERENCE ON EMERGING ELEARNING TECHNOLOGIES AND APPLICATIONS, 9., 2011, Stará Lesná, Eslováquia. Conference Proceedings. Stará Lesná: IEEE, 2011. p. 189 - 194. Disponível em: 
<http://ieeexplore.ieee.org/xpl/articleDetails.jsp?arnumber=6112613>. Acesso em: 06 jul. 2016.

AMANTE, Lúcia; MORGADO, Lina. Metodologia de concepção e desenvolvimento de aplicações educativas: o caso dos materiais hipermídia. Discursos, Lisboa, 3. Série, p. 27-44, jun. 2001. Número Especial. Disponível em:

<http://repositorioaberto.uab.pt/bitstream/10400.2/4348/1/L\%C3\%BAcia\%20Amante_Lina \%20Morgado.pdf>. Acesso em: 22 mar. 2016.

ANDERSON, Terry; DRON, Jon. Three generations of distance education pedagogy. Irrodl, Athabasca, v. 12, n. 3, p. 1-18, mar. 2011. Disponível em <http://www.irrodl.org/index.php/irrodl/article/view/890/1826>. Acesso em: 31 out. 2016.

AUSUBEL, David P.. Aquisição e retenção de conhecimentos: uma perspectiva cognitiva. Lisboa: Plátano Edições Técnicas, 2003.

BATES, Anthony William. Teaching in a digital age: guidelines for designing teaching and learning for a digital age. Vancouver: BCcampus, 2015. (BC Open Textbook Project). Disponível em: <https://opentextbc.ca/teachinginadigitalage/>. Acesso em: 04 jul. 2016.

BEHAR, Patricia Alejandra. Modelos pedagógicos em educação a distância. In: BEHAR, Patricia Alejandra (org.). Modelos pedagógicos em educação a distância. Porto Alegre: Artmed, 2009. Cap. 1. p. 15-32.

BENITE, Anna M. Canavarro; BENITE, Cláudio R. Machado; SILVA FILHO, Supercil Mendes da. Cibercultura em Ensino de Química: Elaboração de um Objeto Virtual de Aprendizagem para o Ensino de Modelos Atômicos. Química Nova na Escola, São Paulo, v. 33, n. 2, p.71-76, mai. 2011. Disponível em: <http://qnesc.sbq.org.br/online/qnesc33_2/01-EQM3010.pdf>. Acesso em: 10 fev. 2016.

BROGAN, Donna R.; KUTNER, Michael H. Comparative analysis of pretest-posttest research designs. The American Statician. [S.I.], v. 34, n. 4, p. 229-232, nov. 1980. Disponível em:

<http://140.116.183.121/ sheu/xpDesign/Articles/pretestPosttestDesigns.pdf>. Acesso em: 19 jul. 2016.

BROWN, Theodore L. et al. Química: a ciência central. 9. ed. São Paulo: Prentice Hall, 2005. 972 p.

BUSARELLO, Raul Inácio; ULBRICHT, Vania Ribas; FADEL, Luciane Maria. A gamificação e a sistemática de jogo: conceitos sobre a gamificação como recurso motivacional. In: FADEL, Luciane Maria et al. Gamificação na Educação. São Paulo: Pimenta Cultural, 2014. Cap. 2, p. 300. 
CHANG, Raymond. Química geral: conceitos essenciais. 4. ed. São Paulo: McGraw-Hill, 2006.

DUARTE, David Wesley Amado; ESMERALDO, Guilherme Álvaro Rodrigues Maia; ABREU, João Alberto Brito. Avaliação 2.0: Utilizando a Colaboração na Construção de um Material Didático por Alunos de Graduação. Conex. Ci. e Tecnol., Fortaleza, v. 9, n. 2, p. 43-49, 2015. Disponível em: < http://conexoes.ifce.edu.br/index.php/conexoes/article/view/705>. Acesso em: 06 jul. 2016.

FILATRO, Andrea. As teorias pedagógicas fundamentais em EAD. In: LITTO, Fredric Michael; FORMIGA, Manuel Marcos Maciel. Educação a distância: o estado da arte. São Paulo: Pearson, 2009. Cap. 14. p. 96-104. (Volume I).

FONSECA, João José Saraiva da. Metodologia da Pesquisa Científica. Fortaleza: UECE, 2002. Apostila do Curso de Especialização em Comunidades Virtuais de Aprendizagem - Informática Educativa. Disponível em:

$<$ http://www.ia.ufrrj.br/ppgea/conteudo/conteudo-2012-

1/1SF/Sandra/apostilaMetodologia.pdf>. Acesso em: 10 jul. 2016.

GIORDAN, Marcelo; GÓIS, Jackson. Telemática Educacional e Ensino de Química: Considerações em Torno do Desenvolvimento de um Construtor de Objetos Moleculares. Revista Latinoamerica de Tecnología Educativa, [S.I.], v. 3, n. 2, p.41-59, 2005. Disponível em: <http://relatec.unex.es/article/view/165>. Acesso em: 10 fev. 2016.

HARVIAINEN, J. Tuomas; MERILÄINEN, Mikko; TOSSAVAINEN, Tommi. The game educator's handbook: revised international edition. [S.I.: s.n.], 2015. Disponível em: <http://www.pelikasvatus.fi/handbook>. Acesso em: 07 jul. 2016.

HOFER, Barbara K.; PINTRICH, Paul R. (Eds.). Personal epistemology: the psychology of beliefs about knowledge and knowing. London: Routledge, 2009.

I-TECH (INTERNATIONAL TRAINING \& EDUCATION CENTER ON HIV). University Of Washington (Ed.). Guião de implementação técnica \#2: orientações para pré-teste e pós-teste. Seattle: I-TECH, 2008. Disponível em:

$<$ http://www.go2itech.org/resources/technical-implementation-

guides/2.TIG_Pre_Pos_Teste_A4.pdf>. Acesso em: 10 jul. 2016.

KUHN, Thomas S.. The Structure of Scientific Revolutions. 2. ed. Chicago: The University Of Chicago, 1970. 
MATTAR, João. Games em educação: como os nativos digitais aprendem. São Paulo: Pearson, 2010.

MELO, Marlene Rios; LIMA NETO, Edmilson Gomes de. Dificuldades de Ensino e Aprendizagem dos Modelos Atômicos em Química. Química Nova na Escola, São Paulo, v. 35, n. 2, p.112-122, mai. 2013. Disponível em:

<http://qnesc.sbq.org.br/online/qnesc35_2/08-PE-81-10.pdf>. Acesso em: 11 fev. 2016.

MORTIMER, Eduardo F.. As chamas e os cristais revisitados: estabelecendo diálogos entre a linguagem científica e a linguagem cotidiana no ensino das ciências da natureza. In: SANTOS, Wildson Luiz P. dos; MALDANER, Otavio Aloisio (orgs.). Ensino de química em foco. ljuí: Unijuí, 2015. Cap. 7, p. 181-208.

OLIVEIRA, Saulo França et al. Softwares de simulação no ensino de atomística: experiências computacionais para evidenciar micromundos. Química Nova na Escola, São Paulo, v. 35, n. 3, p.147-151, ago. 2013. Disponível em:

<http://qnesc.sbq.org.br/online/qnesc35_3/02-EQM-29-12.pdf>. Acesso em: 10 fev. 2016.

OTSUKA, Joice et al. Desenvolvimento de jogos educacionais abertos. In: CONGRESSO BRASILEIRO DE INFORMÁTICA NA EDUCAÇÃO, 1., 2012, Rio de Janeiro. Anais do CBIE. Porto Alegre: SBC, 2012. v. 4, p. 1 - 10. Disponível em: <http://brie.org/pub/index.php/wcbie/article/view/1892>. Acesso em: 09 jul. 2016.

PESQUISA Game Brasil 2016. São Paulo: ESPM, 2016. 70 p. Disponível em: <http://www.pesquisagamebrasil.com.br/pesquisa-2016>. Acesso em: 04 nov. 2016.

RELATÓRIO Final: Mapeamento da indústria brasileira e global de jogos digitais. Mapeamento da indústria brasileira e global de jogos digitais. São Paulo: BNDES, 2014. Disponível em:

<http://www.bndes.gov.br/SiteBNDES/bndes/bndes_pt/Galerias/Arquivos/conhecimento/s eminario/seminario_mapeamento_industria_games042014_Relatorio_Final.pdf >. Acesso em: 05 nov. 2016.

ROLANDO, Luís Gustavo R. et al. Integration between Internet and Chemistry Teaching Practice. Rev. Virtual Quim., São Paulo, v. 7, n. 3, p. 864-879, 2015. DOI: 10.5935/19846835.20150044. Disponível em: <http://rvq.sbq.org.br/index.php/rvq/article/view/898>. Acesso em: 06 jul. 2016.

ROQUE, Nídia Franca; SILVA, José Luis P. B.. A linguagem Química e o ensino da Química orgânica. Química Nova, São Paulo, v. 31, n. 4, p.921-923, jul./ago. 2008. Disponível em: <http://quimicanova.sbq.org.br/imagebank/pdf/Vol31No4_921_33ED08026.pdf>. Acesso em: 21 set. 2015. 
SANTANA, Eliana Moraes de; REZENDE, Daisy de Brito. O uso de jogos no ensino e aprendizagem de Química: uma visão dos alunos do 9 ano do ensino fundamental. In: ENCONTRO NACIONAL DE ENSINO DE QUÍMICA, 14., 2008, Curitiba. Anais... Curitiba: UFPR, 2008. p. 1 - 10. Disponível em:

<http://www.cienciamao.usp.br/dados/eneq/_ousodejogosnoensinoeapre.trabalho.pdf>. Acesso em: 22 abr. 2016.

SANTOS, Ronnie E. S. et al. Contribuições da Comunidade Brasileira de Informática na Educação para o Ensino de Química. Workshop de Informática na Escola, 20., Anais do WIE 2014. Dourados (Mato Grosso do Sul): SBC, 2014, p. 136 - 145. Disponível em: <http://www.br-ie.org/pub/index.php/wie/article/view/3092>. Acesso em 06 jul. 2016.

SANTOS, Wildson Luiz Pereira dos; SCHENETZLER, Roseli Pacheco. Educação em Química: compromisso com a cidadania. 4. ed. rev. atual. ljuí: Ed. Unijuí, 2010.

SATO, Adriana Kei Ohashi; CARDOSO, Marcos Vinicius. Além do gênero: uma possibilidade para a classificação de jogos. In: SBGAMES, 7., 2008, Belo Horizonte. Proceedings of SBGAMES'08: art \& design track. Belo Horizonte: SBC, 2008. p. 54 - 63. Disponível em: <http://www.sbgames.org/papers/sbgames08/Proceedings-SBGames-GC2008-Final-CD.pdf>. Acesso em: 04 nov. 2016.

SCHNETZLER, Roseli P.. A pesquisa em ensino de Química no Brasil: conquistas e perspectivas. Química Nova, São Paulo, v. 25, n. 1, p.14-24, 2002. Disponível em: <http://quimicanova.sbq.org.br/imagebank/pdf/SBQ-

25AnosVol25Sup1Especial_14_03.pdf>. Acesso em: 07 mar. 2016.

SENDLINGER, S. C. et al. Transforming Chemistry Education through Computational Science. Comput. Sci. Eng., [S.I.], v. 10, n. 5, p. 34-39, 2008. Institute of Electrical \& Electronics Engineers (IEEE). DOI: 10.1109/mcse.2008.124. Disponível em: <http://ieeexplore.ieee.org/xpl/articleDetails.jsp?arnumber=4604503>. Acesso em: 06 jul. 2016.

SIEMENS, George. Conectivismo: uma teoria da aprendizagem para a era digital. 2004. Disponível em: <http://usuarios.upf.br/ teixeira/livros/conectivismo[siemens].pdf>. Acesso em: 04 jul. 2016.

SILVA, Glenda Rodrigues da; MACHADO, Andréa Horta; SILVEIRA, Katia Pedroso. Modelos para o Átomo: Atividades com a Utilização de Recursos Multimídia. Química Nova na Escola, São Paulo, v. 37, n. 2, p.106-111, mai. 2015. Disponível em:

<http://qnesc.sbq.org.br/online/qnesc37_2/06-EQM-83-13.pdf>. Acesso em: 10 fev. 2016.

TALIB, Othman et al. Implementing the SEA Application for Organic Chemistry: ArrowPushing versus Electron-Moving Techniques. INFORMATION TECHNOLOGY BASED HIGHER EDUCATION AND TRAINING (ITHET), 2014, York (England). Conference 
Proceedings. York: IEEE, 2014. p. 1-5. DOI: 10.1109/ITHET.2014.7155719. Disponível em: <http://ieeexplore.ieee.org/xpl/articleDetails.jsp?arnumber=7155719>. Acesso em: 08 jul. 2016.

TAVARES, Romero. Aprendizagem significativa e o ensino de ciências. Ciências e Cognição, Rio de Janeiro, v. 13, n. 1, p.94-100, 31 mar. 2008. Disponível em: <http://cienciasecognicao.tempsite.ws/revista/index.php/cec/article/view/687/464>. Acesso em: 07 mar. 2016.

TELES, Rennan Rodrigues Isidio; SILVA, Igor William Pessoa da; DUARTE, David Wesley Amado. Um levantamento bibliográfico sobre o uso das TICs para o ensino de Química. In: SEMANA DE INICIAÇÃO CIENTÍFICA DE INFORMÁTICA, 1., 2016, Crato. Anais... Crato, CE: IFCE, 2016.

TORI, Romero. Educação sem distância: as tecnologias interativas na redução de distâncias em ensino e aprendizagem. São Paulo: Editora SENAC, 2010.

VASCONCELOS, Flávia Cristina Gomes Catunda; ARROIO, Agnaldo. Explorando as Percepções de Professores em Serviço Sobre as Visualizações no Ensino de Química. Quím. Nova, São Paulo, v. 36, n. 8, p. 1242-1247, mai. 2013. Disponível em: <http://quimicanova.sbq.org.br/imagebank/pdf/Vol36No8_1242_24-ED12968.pdf>. Acesso em: 06 jul. 2016.

WARMAN, Peter. Newzoo Games (Ed.). 2016 Global Games Market Report: an overview of trends \& insights. San Francisco: Newzoo, 2016. Disponível em: $<$ https://newzoo.com/insights/trend-reports/2016-global-mobile-market-report/>. Acesso em: 04 nov. 2016.

ZICHERMANN, Gabe; CUNNINGHAM, Cristopher. Gamification by design: implementing game mechanics in web and mobile apps. Sebastopol: O'reilly Media, 2011. 